\title{
Hepatitis autoinmune y cáncer de colon: una asociación inusual. Caso clínico y revisión de la literatura
}

\section{A case of an unusual association of autoimmune hepatitis and colon cancer: report and literature review}

Lázaro Antonio Arango M., ${ }^{1}$ Claudia Patricia Díaz T., ${ }^{2}$ Mario Andrés Jaramillo, ${ }^{3}$ Dínimo José Bolívar S., Mauricio Osorio C., ${ }^{5}$ Alba Ruth Cobo, ${ }^{6}$ Carlos Andrés Caicedo Q. ${ }^{7}$

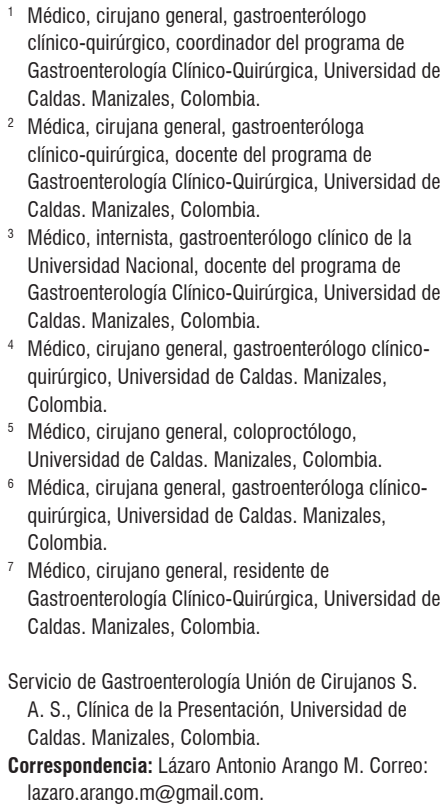

3 Médico, internista, gastroenterólogo clínico de la Universidad Nacional, docente del programa de Gastroenterología Clínico-Quirúrgica, Universidad de Caldas. Manizales, Colombia.

${ }^{4}$ Médico, cirujano general, gastroenterólogo clínicoquirúrgico, Universidad de Caldas. Manizales, Colombia.

5 Médico, cirujano general, coloproctólogo, Universidad de Caldas. Manizales, Colombia.

${ }^{6}$ Médica, cirujana general, gastroenteróloga clínicoquirúrgica, Universidad de Caldas. Manizales, Colombia.

7 Médico, cirujano general, residente de Gastroenterología Clínico-Quirúrgica, Universidad de Caldas. Manizales, Colombia.

Servicio de Gastroenterología Unión de Cirujanos S. A. S., Clínica de la Presentación, Universidad de Caldas. Manizales, Colombia.

Correspondencia: Lázaro Antonio Arango M. Correo: lazaro.arango.m@gmail.com.

Fecha recibido: $14-08-17$

Fecha aceptado: $13-04-18$

\begin{abstract}
Resumen
Introducción: la hepatitis autoinmune idiopática (HAl) es una enfermedad crónica que predomina en mujeres, con episodios de actividad y remisión, favoreciendo la fibrosis hepática. El $40 \%$ de los pacientes presenta historia familiar de enfermedades autoinmunes. Al parecer, es mediada por la interacción antígeno-anticuerpo; sin embargo, su causa es desconocida. Se conoce la asociación frecuente de HAI con cáncer hepatobiliar; menos frecuente con linfomas, cáncer de piel y cáncer de colon; y casi inexistente con síndromes hereditarios de cáncer de colon. Este caso debutó con HAl y sangrado rectal causado por poliposis adenomatosa familiar (PAF) y adenocarcinoma de colon sigmoide.

Caso clínico: mujer de 51 años con HAl de 1 año de evolución manejada con prednisolona y azatioprina. Se realizó una colonoscopia total por anemia en la que se encontraron múltiples pólipos entre 5 y $10 \mathrm{~mm}$ y 1 de $30 \mathrm{~mm}$ sésil, ulcerado, en colon sigmoide. Se realizó una polipectomía endoscópica múltiple que reportó un adenoma tubulovelloso con displasia de alto y bajo grado en varios pólipos y un adenocarcinoma de bajo grado en el pólipo del sigmoide. Los estudios de extensión fueron negativos para metástasis. Se realizó una repleción nutricional prequirúrgica, luego una colectomía subtotal y una procto-ileoanastomosis con ileostomía de protección. La patología de pieza quirúrgica mostró un adenocarcinoma de colon de bajo grado y adenomas tubulares y tubulovellosos con displasias de alto y bajo grado.

Discusión y conclusiones: La asociación de HAI con PAF y cáncer colorrectal (CC) es infrecuente. Es conocida la correlación de HAI con cáncer hepatobiliar (asociado con cirrosis), linfomas, cáncer de piel y otros desórdenes autoinmunes. El pronóstico es malo y no puede establecerse una correlación clara con moduladores inmunes.
\end{abstract}

\section{Palabras clave}

Hepatitis autoinmune, malignidades, neoplasias extrahepáticas, poliposis adenomatosa, carcinoma colorrectal.

\section{INTRODUCCIÓN}

La hepatitis autoinmune idiopática (HAI) tiene varias asociaciones: por ejemplo, predispone el desarrollo de cirrosis hepática. Esta última es un factor establecido para cáncer hepatocelular ( $\mathrm{CHC})$, con una incidencia anual entre 3\%-9\%; es inducida especialmente por las hepatitis crónicas virales B y C (1). Hasta un tercio de los pacientes al momento del diagnóstico de HAI ya han desarrollado cirrosis (2). 


\begin{abstract}
Introduction: Idiopathic autoimmune hepatitis $(\mathrm{IAH})$ is a chronic disease that occurs predominately in women, has episodic activity and remission, and favors hepatic fibrosis. Forty percent of patients have family histories of autoimmune diseases. It is apparently mediated by antigen-antibody interaction, but its causes are unknown. IAH is frequently associated with hepatobiliary cancer, less frequently with lymphomas, skin cancer and colon cancer and very rarely with hereditary colon cancer syndrome. This case debuted IAH and rectal bleeding caused by familial adenomatous polyposis (FAP) and adenocarcinoma of the sigmoid colon.

Clinical case: The patient was a 51-year-old woman who had had IAH for one year which had been managed with prednisolone and azathioprine. A total colonoscopy, performed because of anemia, found multiple polyps that measured 5 and $10 \mathrm{~mm}$ and one ulcerated $30 \mathrm{~mm}$ sessile polyp in the sigmoid colon. A multiple endoscopic polypectomy revealed a tubulovillous adenoma with high and low grade dysplasia in several polyps and a low grade adenocarcinoma in the sigmoid polyp. Tests and examinations for metastasis were negative. Following presurgical nutritional repletion, a subtotal colectomy was performed and an ileal pouch-anal anastomosis with protective ileostomy was created. The pathology of the surgical specimen showed low grade adenocarcinoma of the colon and tubular and tubulovillous adenomas with high and low grade dysplasia.

Discussion and conclusions: Association of IAH with familial adenomatous polyposis (FAP) and colorectal cancer (CC) occurs infrequently although associations of IAH with hepatobiliary cancer associated with cirrhosis, lymphomas, skin cancer and other autoimmune disorders are well-known. The prognosis is bad and no clear correlation with immune modulators can be established.
\end{abstract}

\title{
Keywords
}

Autoimmune hepatitis, malignancies, extra-hepatic neoplasms, adenomatous polyposis, colorectal carcinoma.

La HAI se puede asociar con varias enfermedades autoinmunes, esta relación se encuentra bien establecida y el riesgo de malignidad con el tratamiento inmunosupresor es una preocupación fundamental (3). La asociación de HAI con poliposis adenomatosa familiar (PAF) o cáncer colorrectal (CC) es extremadamente infrecuente, por lo que se describe un caso clínico y se revisa la escasa literatura disponible.

\section{CASO CLÍNICO}

La paciente es una mujer de 51 años con ictericia intermitente de 18 meses de evolución, prurito, coluria y rectorragia, sin antecedentes de importancia. No refirió medicamentos hepatotóxicos. En el examen clínico se encontró con ictericia, sin osteomialgias o pérdida de peso, abdomen normal; antígeno carcinoembrionario $1,57 \mathrm{ng} / \mathrm{mL}(0-10)$; serología para hepatitis B y $\mathrm{C}$ negativas; $\gamma$-glutamiltransferasa (GGT) $460 \mathrm{UI} / \mathrm{L}$; bilirrubina total $3,7 \mathrm{mg} / \mathrm{dL}$; transaminasa glutámico oxalacética (TGO) 116, transaminasa glutámico pirúvica (TGP) 119 y albúmina $3,8 \mathrm{mg} / \mathrm{dL}$; hemoglobina $(\mathrm{Hb})$ 9,6 mg/dL,; hematócrito (Hto) 32\%; anticuerpos antinucleares (ANA) y anticuerpos antimúsculo liso (ASMA) positivos; anticuerpos antimitocondria negativos (AMA); ultrasonografía abdominal sin lesiones hepáticas ni colelitiasis; vía biliar normal; biopsia hepática con hepatitis de interface de actividad moderada, sin hepatitis lobulillar ni necrosis y sin presencia de granulomas.
Se hizo el diagnóstico de HAI y se inició una terapia inmunomoduladora con prednisolona y azatioprina. Debido a la anemia se ordenó una endoscopia que reportó esofagitis grado B de los Ángeles y gastritis erosiva antral, y una colonoscopia con 25 a 30 pólipos de diámetros entre 0,5 a $1 \mathrm{~cm}$ a lo largo de todo el colon. Se realizaron biopsias al azar de numerosos pólipos así: 2 pólipos de colon derecho con adenoma tubulovelloso y displasia de alto grado; 2 pólipos de colon transverso y descendente con adenoma tubulovelloso con displasia de bajo grado; lesión polipoide en colon sigmoide a los $30 \mathrm{~cm}$ del reborde anal de $3 \mathrm{~cm}$, ulcerada, positiva para adenocarcinoma de colon de bajo grado. La radiografía de tórax y la tomografía axial computarizada (TAC) de abdomen resultaron normales. Se realizó una repleción nutricional prequirúrgica y posteriormente una laparotomía en la que se observó el hígado sin nódulos, ni ascitis ni siembras peritoneales. Se realizó una colectomía subtotal con procto-ileoanastomosis terminolateral e ileostomía de protección (Figura 1).

La patología mostró múltiples formaciones polipoides sésiles en toda la extensión del colon, con medidas promedio de $0,8 \times 0,5 \mathrm{~cm}$ y $2 \times 1,5 \mathrm{~cm}$, fue positivo para adenomas tubulovellosos con displasia de bajo y alto grado. En el colon sigmoide se presentó una lesión polipoide ulcerada positiva para adenocarcinoma de colon de bajo grado, bien diferenciado, con infiltración de capa muscular propia e invasión linfovascular, y serosa libre de tumor. Se observó un pólipo de $1,3 \times 0,3 \mathrm{~cm}$ en recto superior y un adenoma 
tubular con displasia de alto grado, negativo para malignidad. Hubo $3 / 13$ ganglios positivos con bordes de resección libres y T3N1bM0.

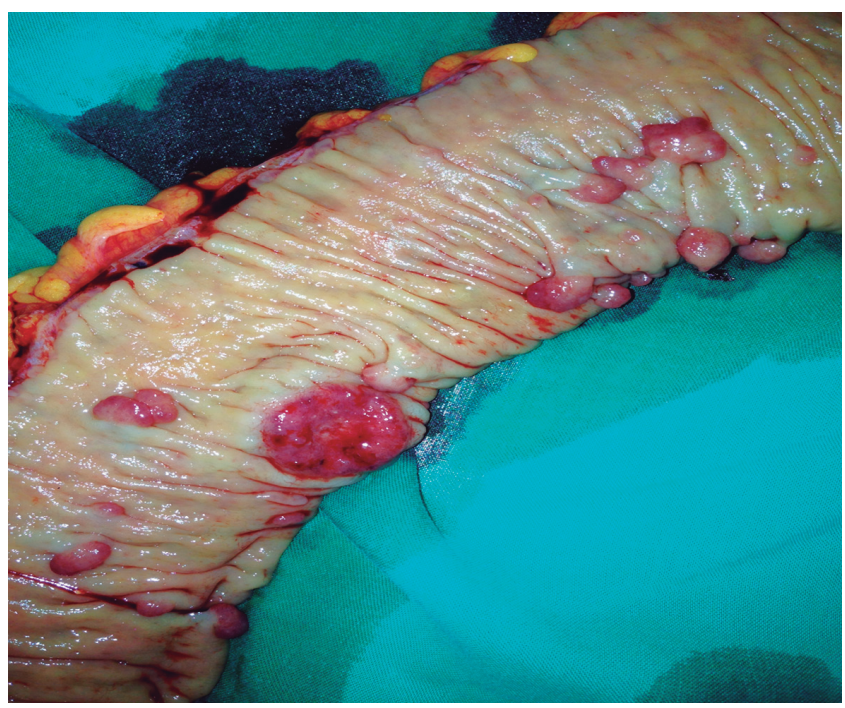

Figura 1. Espécimen quirúrgico en el que se observa la pieza colónica con múltiples pólipos en toda su extensión.

\section{DISCUSIÓN}

La frecuencia de malignidad extrahepática en HAI es del $5 \%$ con incidencia estimada de 1 caso por cada 194 pacientes/año. La probabilidad de tumores extrahepáticos después de 10 años es del $3 \%$ y el riesgo es 1,4 veces mayor que en la población general. Los mecanismos patogénicos que promueven el desarrollo de neoplasias extrahepáticas en la HAI son desconocidos y no se asocian con cirrosis (4).

Las malignidades extrahepáticas desarrolladas antes y después del diagnóstico de HAI se originan en varios órganos incluidos las vejiga, sangre, mama, cérvix, tejido linfoide, piel, timo, tejidos blandos y estómago (5).

Werner y colaboradores evaluaron a 473 pacientes con HAI entre 1990 y 2003 presentes en el registro nacional de cáncer de Suecia y encontraron 108 tumores, 39 benignos y 69 malignos; 25 fueron prevalentes (antes del diagnóstico de HAI) y 44 incidentales (después del diagnóstico). Esta cohorte analizó el riesgo para cáncer hepatobiliar, piel, linfoma no hodgkiniano, colon y recto, entre otros. Se encontraron 4 pacientes con $\mathrm{CC}$, con riesgo ligeramente elevado con riesgo de incidencia estándar (RIE) de 4,23 e intervalo de confianza (IC) $95 \%$ de 1,1 a 10,8; ninguno tenía historia de enfermedad inflamatoria intestinal. A diferencia del alto riesgo para cáncer hepatobiliar, linfoma y cáncer de piel (RIE: 8,4, IC 95\%: 2,66-19,2; RIE: 5,91, IC 95\%: 1,9-13,7; y RIE: 6,1, IC 95\%: 2,47-12,7), respectivamente.
La HAI es una enfermedad progresiva poco frecuente que puede inducir cirrosis y $\mathrm{CHC}$; sin embargo, su relación es menos obvia y subestimada que la bien conocida cirrosis hepática y CHC por hepatitis B o C. El riesgo de padecer cáncer luego del diagnóstico de HAI se eleva. Únicamente 4 de 473 pacientes con HAI del registro nacional de cáncer de Suecia presentaron CC (6).

Los tumores asociados con la inmunosupresión crónica después del trasplante de órgano (por ejemplo, cáncer de piel) se correlacionan con efectos inducidos por fármacos sobre mecanismos inmunológicos involucrados en la vigilancia tumoral y el control de infecciones. Un estudio sueco de 634 pacientes con HAI mostró la asociación conocida de $\mathrm{CHC}$ y reconoció el cáncer de piel tipo no melanoma como la presentación extrahepática tumoral más frecuente (23 casos), seguido por el linfoma no hodgkiniano (7). En la HAI, la duración y el grado de la terapia inmunosupresora no pueden ser descartados como factores de riesgo para la aparición de tumores (8).

La supresión de la función de linfocitos y mecanismos de vigilancia tumoral mediados por citocinas, la alteración de la apoptosis y crecimiento tumoral a través de vías de señalización celular (9), el daño directo del ADN y sus mecanismos de reparación, la menor expresión de genes supresores de tumor, la mala regulación del ciclo celular (10), la severidad de la enfermedad autoinmune y el tipo y dosis de los fármacos administrados son algunos efectos especulativos que inducirían los medicamentos para desarrollar cáncer en pacientes con $\mathrm{HAI}(6)$.

Es interesante comparar los riesgos de PAF o CC con otras enfermedades biliares/hepáticas autoinmunes. En una cohorte sueca de 604 pacientes con colangitis esclerosante primaria entre 1970 y 1998, el CHC se observó en 12 pacientes con enfermedad inflamatoria intestinal concomitante con un RIE de 10,3 (IC 95\%: 5,3-18,1) (11).

En un estudio escocés de 85 pacientes con cirrosis biliar primaria, de 10 pacientes que presentaron malignidades extrahepáticas solo 1 presentó CC (12). En un metaanálisis realizado por Liang y colaboradores con 16300 pacientes con colangitis esclerosante primaria, encontraron que el riesgo de cáncer general y $\mathrm{CHC}$ es mayor en comparación con la población general, pero no con otros tipos de cáncer. 50 casos de cáncer de colon y 23 con cáncer de recto fueron registrados con un RIE de 1,13 (IC 95\%: -0,26 a -2,52) y RIE: 1 (IC 95\%: 0,56 a 1,43), respectivamente (13).

Después de comparar otras enfermedades hepáticas autoinmunes, se puede concluir que existe un incremento similar en el riesgo de cáncer hepático y extrahepático, lo que sugiere la necesidad de crear programas de vigilancia estrictos y, aunque la asociación de HAI y CC no es estadística, se requieren estudios que permitan evaluar esta relación. 
La evidencia disponible de pacientes con HAI y tumores extrahepáticos se basa en su mayoría en tumores no gastrointestinales. Entre los gastrointestinales, el CC es el más frecuente. El tratamiento debe ser acorde a cada patología y puede incluir resección, radioterapia o quimioterapia (8). La terapia inmunosupresora debe ajustarse $y$, aunque estas respuestas no se han documentado con HAI, en lo posible se debe reducir la dosis de medicación o sustituirla por un fármaco alterno. La acción apropiada es suspender la azatioprina y otros inmunosupresores no estándar (inhibidores de calcineurina y micofenolato mofetilo), y controlar la actividad inflamatoria hepática con el aumento de la dosis de corticosteroides (6). Incluso en pacientes postransplantados hay aumento en la aparición de CC para aquellos que tienen terapia inmunomoduladora con tiopurinas más esteroides, así como incidencia mayor para linfoma y sarcoma; los pacientes tratados con ciclosporina no tienen un aumento del riesgo (14).

A pesar de la resección quirúrgica del adenocarcinoma de colon en nuestro caso, existe un riesgo elevado para las neoplasias adicionales; por esta razón debe realizarse un seguimiento endoscópico, imagenológico y con marcadores tumorales. La mejoría en métodos diagnósticos, el creciente número de reportes de HAI y malignidades extrahepáticas, los nuevos tratamientos farmacológicos moleculares y de intervención celular que pueden deteriorar la respuesta inmunológica contra el cáncer, y el incremento de la supervivencia en HAI, cirrosis y cáncer extrahepático justifican un cambio de mentalidad y toma de conciencia acerca de este tipo de patologías.

\section{CONCLUSIÓN}

Los pacientes con HAI presentan riesgo aumentado ya conocido de CHC y neoplasias extrahepáticas. El mecanismo patogénico no es del todo conocido. Es poca la literatura disponible que relacione la HAI con el CC, y ninguna la que relaciona la HAI con la PAF. Existe la necesidad de crear programas de evaluación y vigilancia más estrictos que permitan predecir el comportamiento biológico, el manejo y supervivencia a este tipo de tumores.

\section{REFERENCIAS}

1. Velázquez RF, Rodríguez M, Navascués CA, et al. Prospective analysis of risk factors for hepatocellular carcinoma in patients with liver cirrhosis. Hepatology. 2003;37(3):520-7. doi: 10.1053/jhep.2003.50093.
2. European Association for the Study of the Liver. EASL Clinical Practice Guidelines: Autoimmune hepatitis. J Hepatology. 2015;63(4):971-1004. doi: 10.1016/j. jhep.2015.06.030.

3. Smedby KE, Baecklund E, Askling J. Malignant lymphomas in autoimmunity and inflammation: a review of risks, risk factors, and lymphoma characteristics. Cancer Epidemiol Biomarkers Prev. 2006;15(11):2069-77. doi: 10.1158/1055-9965.EPI-06-0300.

4. Wang KK, Czaja AJ, Beaver SJ, et al. Extrahepatic malignancy following long-term immunosuppressive therapy of severe hepatitis B surface antigen-negative chronic active hepatitis. Hepatology. 1989;10(1):39-43. doi: 10.1002/ hep. 1840100110.

5. Czaja AJ. Hepatocellular carcinoma and other malignancies in autoimmune hepatitis. Dig Dis Sci. 2013;58(6):1459-76. doi: 10.1007/s10620-012-2525-5.

6. Werner M, Almer S, Prytz H, et al. Hepatic and extrahepatic malignancies in autoimmune hepatitis. A long-term followup in 473 Swedish patients. J Hepatol. 2009;50(2):388-9. doi: 10.1016/j.jhep.2008.08.022.

7. Komaki Y, Komaki F, Micic D, et al. Risk of colorectal cancer in chronic liver diseases: a systematic review and metaanalysis. Gastrointest Endosc. 2017;86(1):93-104.e5. doi: 10.1016/j.gie.2016.12.009.

8. Penn I. Post-transplant malignancy: the role of immunosuppression. Drug Saf. 2000;23(2):101-13. doi: 10.2165/00002018-200023020-00002.

9. Han W, Soltani K, Ming M, et al. Deregulation of XPC and CypA by cyclosporin A: an immunosuppression-independent mechanism of skin carcinogenesis. Cancer Prev Res (Phila). 2012;5(9):1155-62. doi: 10.1158/1940-6207. CAPR-12-0185-T.

10. Strickland FM, Kripke ML. Immune response associated with nonmelanoma skin cancer. Clin Plast Surg. 1997;24(4):637-47.

11. Bergquist A, Ekbom A, Olsson R, et al. Hepatic and extrahepatic malignancies in primary sclerosing cholangitis. J Hepatol. 2002;36(3):321-7. doi: 10.1016/S01688278(01)00288-4

12. Mills PR, Boyle P, Quigley EM, et al. Primary biliary cirrhosis: an increased incidence of extrahepatic malignancies? J Clin Pathol. 1982;35(5):541-3. doi: 10.1136/jcp.35.5.541.

13. Liang Y, Yang Z, Zhong R. Primary biliary cirrhosis and cancer risk: a systematic review and meta-analysis. Hepatology. 2012;56(4):1409-17. doi: 10.1002/hep.25788.

14. Danielsson Borssén $\AA$, Almer S, Prytz H, et al. Hepatocellular and extrahepatic cancer in patients with autoimmune hepatitis - a long-term follow-up study in 634 Swedish patients. Scand J Gastroenterol. 2015;50(2):217-23. doi: $10.3109 / 00365521.2014 .983154$. 